\title{
Issues Surrounding Teaching Linear Algebra
}

\author{
Avi Berman and Kazuyoshi Okubo
}

\section{Report}

Linear Algebra is one of the most important courses in the education of mathematicians, scientists, engineers and economists. DG 3 was organized by The Education Committee of International Linear Algebra Society (ILAS) in order to give mathematicians and mathematics educators the opportunity to discuss several issues on teaching and learning Linear Algebra including motivation, challenging problems, visualization, learning technology, preparation in high school, history of Linear algebra and research topics at different levels. Some of these problems were discussed. Around 50 participants participated in the discussion.

\section{Motivation}

The interest in learning linear algebra can be motivated by real life (high tech) applications and by challenging problems. The following examples were mentioned:

Organizers Co-chairs: Avi Berman (Israel), Kazuyoshi Okubo (Japan); Team Members: Steven Leon (USA), Sepideh Stewart (New Zealand), Sang-Gu Lee (Korea), David Strong (USA); Liaison IPC Member: K. (Ravi) Subramaniam.

\footnotetext{
A. Berman ( $\square)$

Israel Institute of Technology, Haifa, Israel

e-mail: berman@technion.ac.il

K. Okubo

Hokkaido University of Education, Sapporo, Japan

e-mail: okubo.kazuyoshi@s.hokkyodai.ac.jp

(C) The Author(s) 2015 
Google's Page Rank; Edge detection methods; Neural networks; Problems in graph theory; Properties of the Fibonacci sequence; Computer games - "Fiver" (see http://www.math.com/students/puzzles/fiver/fiver.html), Another popular game that can be solved by linear algebra (see http://matrix.skku.ac.kr/bljava-v1/Test.html).

\section{Technology}

Sang-Gu Lee from Korea described the work on Sage. He talked about what can be done in Mobile learning Environment and mentioned his coming regular lecturer on Mobile LA. He mentioned Bill Barton' words on Monday "We know that Education using ICT will improve the quality of Math Education. But it is clear that we are not THERE yet" (ICT Revolution in Math Education). http://www. sciencetimes.co.kr/article.do?todo $=$ view \&atidx $=0000063949$. Avi Berman from Israel described the use of clickers to promote the students' involvement and the communication between them and the professors.

J. L. Dorrier from France raised a question "Is it a good idea to use computers?" Sang-Gu said "If we do it properly, there is no reason for not using technology for education. I am used to teach and discuss in a traditional way, but I encourage our students to use whatever they can use for better understanding of Linear Algebra." Dorrier said that he prefers to wait with the use of computers to a later stage. Other participants said that they use Maple from the beginning of the course. Ludwig Paditz from Germany pointed out that technology sometimes gives incorrect results. "Modern calculators (CAS) resolve some problems but sometimes incorrect". It is important that students check if the computed results make sense.

\section{Understanding}

Megan Wawro from the USA described her research with Chris Rasmussen on how students engage with eigenvalue-eigenvector system making connections with functions.

Sepideh Stewart (New Zealand, USA) described her PhD thesis on teaching and learning Linear Algebra. She made a framework using Tall's embodied symbolic and formal words of mathematical thinking in conjunction with Ed Dubinsky's APOS theory. She found that the majority of the students were comfortable in the symbolic world but struggled with formal definitions and theorems. She also found that embodied (giving body to an abstract idea) thinking helped some students to have a better grasp of Linear Algebra. Her thesis is available on the web. 


\section{Teaching Mathematics and Engineering Students}

Saeja Kim at U Mass, Dartmouth said she is not in favor of introducing Linear Algebra in an abstract way. She suggested to start with linear equations pointing out that most students are struggling with even the basics ideas. Avi said he also starts with linear equations but quickly moves into more abstract theory. Dorrier said that Linear Algebra is not about solving systems. The main thing is having mental views. He believes that abstraction ability of math majors should be developed. Avi said that at the Technion this is done also for students of electrical engineering and computer science.

Michelle Zandieh (Arizona) and many other participants emphasized the importance of Geometry and Visualization.

Chris Rasmussen (San Diego) asked how the differential equations and the linear algebra courses can be combined. The question was answered by a presentation by Karsten Schmidt from Denmark titled: "Revising the first semester math course for engineering students".

The names of the participants and photos from the two sessions can be found in http://matrix.skku.ac.kr/2014-Album/ICME12-DG3-report-v1.htm.

Open Access This chapter is distributed under the terms of the Creative Commons Attribution Noncommercial License, which permits any noncommercial use, distribution, and reproduction in any medium, provided the original author(s) and source are credited. 